\title{
Coincident Neutrino and Gamma-Ray Emission from
}

\section{Blazars}

\section{Marcel Schroller, ${ }^{a, b, *}$ Julia Becker Tjus, ${ }^{a, b}$ Patrick Reichherzer, ${ }^{a, b, c}$ Ilja Jaroschewski, ${ }^{a, b}$ Mario Hörbe ${ }^{a . b}$ and Wolfgang Rhode ${ }^{b, d}$}

${ }^{a}$ Ruhr-University Bochum, Theoretische Physik IV: Plasma-Astroteilchenphysik, Universitätsstrasse 150, 44801 Bochum, Germany

${ }^{b}$ Ruhr Astroparticle And Plasma Physics Center (RAPP Center)

${ }^{c}$ University Paris-Saclay, IRFU, CEA, F-91191 Gif-sur-Yvette, Paris, France

${ }^{d}$ TU Dortmund, Experimentelle Physik $V$, August-Schmidt-Strasse 1, 44221 Dortmund, Germany

E-mail: marcel.schroller@ruhr-uni-bochum.de

Active galactic nuclei (AGN), and the accompanied AGN jets, are among the most fascinating and luminous objects in the observable Universe. Both their active cores and their jets are candidates for the acceleration of cosmic rays, and subsequent production of gamma rays and neutrinos. A deepened understanding of the processes related to jets will not only fuel the field of high energy cosmic rays, but will also give insights in fundamental plasma, astro, and particle physics. The physical and mathematical modeling of an AGN jet is challenging, with ambiguous signatures that need to be understood by numerical simulations of cosmic ray transport and interactions. Based on the work of Hoerbe et al. (MNRAS 2020), a simulation framework for hadronic constituents and their interactions inside of a plasmoid, propagating along the AGN jet axis, was developed. The final goal of the simulation is to give predictions in the context of multi-messenger astrophysics. This talk will present the first results, discuss the question of diffusivity of the particles and examine the scenario, where neutrino and gamma-ray flares coincide.

$37^{\text {th }}$ International Cosmic Ray Conference (ICRC 2021)

July 12th - 23rd, 2021

Online - Berlin, Germany

\footnotetext{
*Presenter
} 


\section{Introduction}

High-energy neutrinos, as measured by the IceCube observatory in the past few years, are tracers of hadronic interactions and thus, can be used to close in on the origin of ultra-high-energy cosmic rays (UHECRs) and their acceleration sites [1,2]. AGN, particularly the AGN-subclass of blazars, are in discussion as potential sources for UHECRs for a long time[3-6]. Recently, it was hypothesized that the production of high-energy neutrinos in hadronic interactions occurs while the gamma-ray activity of the blazar is deficient [13]. This idea is supported by the an anti-correlation between gamma-rays and radio emission at the detection time of the high-energy neutrino [13], while at other times, radio and gamma-rays rather correlate.

Numerical calculations are able to help to disentangle the connections between the individual messengers [8]. For this purpose, the CRPropa-framework ${ }^{1}$ was utilized to simulate the ballistic propagation of high-energy protons along an AGN-jet axis, in contrast to the common approach of a diffusive propagation. Additional modifications were made to model the environment, as well as to include hadronic interactions into the framework ${ }^{2}$. In this article, the numerical setup is briefly presented, in addition to an excerpt of a run-time analysis. Additionally, the generated data are processed to investigate the correlation between secondary gamma-rays and neutrinos.

\section{Simulation Setup}

The simulations have been created by significantly modifying the CRPropa framework[9] to work in a local source environment, in which a plasmoid is assumed at rest and the AGN environment is passing by the plasmoid in relativisitc motion. A final change of reference frame provide the particle spectra in the observer's frame [8]. In this framework, the particles can either be propagated by solving the equation of motion, representing a ballistic approach, or via the solution of the transport equation, implemented into the CRPropa framework by using the method of Stochastic Differential Equations In [8], first results using the ballistic approach for particles with $10^{8} \mathrm{GeV}$ have been presented. The simulation starts with the homogeneous injection of a fixed number $N_{p}$ of protons into a spherical region with radius $R$, hereafter referred to as plasmoid. The energy of the protons will either be monochromatic or power-law-like distributed with spectral index $\alpha_{p}$ and the injection is instantaneous in time at some starting point $t_{0}$.

The plasmoid itself will start propagating at a defined distance $r_{0}$ from the central engine, the SMBH or SMBBH, with bulk Lorentz factor $\Gamma$. A thin accretion disk will illuminate the plasmoid, resembling a thermal black body field and is Doppler de-boosted inside the plasmoid's rest frame. After traveling a pre-defined distance $d=r_{\text {end }}-r_{0}$, the simulation will terminate.

Furthermore, the jet, which is launched from the AGN, will contain a relativistic plasma, hadronic and leptonic, which is permeated by a purely Kolmogorov-turbulent magnetic field with correlation length $l_{c}$ and mean-field strength at the base of the jet, $B_{0}$. The turbulent field is designed to deflect all ambient charged particles in order to isotropize their velocities in a few simulation steps.

Besides the deflection, ambient electrons contribute to the interactions with their isotropic synchrotron emission during their gyration around the magnetic field lines. The hadronic component

${ }^{1}$ CRPropa v3.1 [9]

2https://github.com/Marcel-Schroller/crpropa_AGN_plasmoid.git 


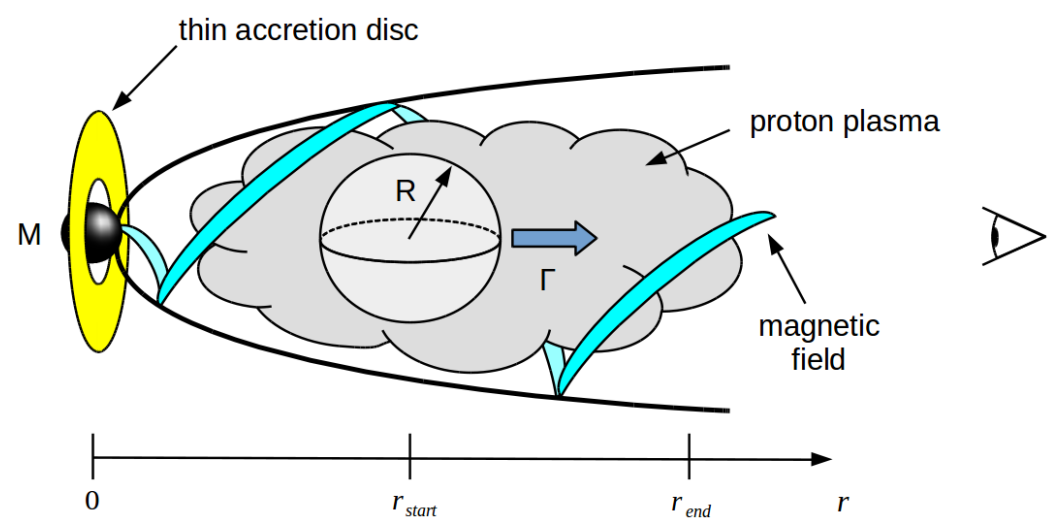

Figure 1: The abstract simulation scheme, with plasmoid of radius $R$, bulk Lorentz factor $\Gamma$, central engine with thin accretion disk as well as the jet structure, depicted as a cone. The jet is assumed to contain a relativistic plasma and a Kolmogorov-turbulent magnetic field. Taken from [8].

of the relativistic plasma will inflict proton-proton scattering inside the plasmoid. Ambient protons are designed as a scalable matter field in the sense of space- and time-dependent interactions. Energetically, the magnet field and ambient plasma are designed to be in equipartition:

$$
U_{B}=\frac{B^{2}}{2 \mu_{0}} \stackrel{!}{=} U_{\text {particles }}=U_{p}+U_{e}
$$

with the vacuum permeability $\mu_{0}$ and the respective particle energy densities $U_{p}$ and $U_{e}$ for protons and electrons.

For a detailed discussion about all scaling functions implied in this model description, see [8], where all definitions are given in the sections 2.1-2.5.

Table 1: Excerpt of the parameter space. Non-essential parameter are omitted because of the limited scope of this article. Please contact the authors for additional information, if desired.

\begin{tabular}{lcc}
\hline Parameter & Symbol & Value \\
\hline Plasmoid Radius & $R$ & $10^{13} \mathrm{~m}$ \\
Initial magnetic field RMS Value & $B_{\mathrm{RMV}}$ & $1 \mathrm{G}$ \\
Correlation length & $l_{c}$ & $R / 100$ \\
Maximum trajectory length & $D_{\max }$ & $1 \mathrm{pc}$ \\
Minimum Candidate energy & $E_{\min }$ & $10^{4} \mathrm{GeV}$ \\
Plasmoid Propagation Start & $r_{\mathrm{start}}$ & $10^{14} \mathrm{~m}$ \\
Plasmoid Propagation End & $r_{\mathrm{end}}$ & $r_{\text {start }}+10 \mathrm{pc}$ \\
Plasmoid Lorentz Factor & $\Gamma$ & 10 \\
Proton Target Density & $n_{0, \text { plasma }}$ & $10^{15} \mathrm{~m}^{-3}$ \\
Electron Minimal Lorentz Factor & $\gamma_{e, \min }$ & 10 \\
Electron Maximal Lorentz Factor & $\gamma_{e, \max }$ & $10^{6}$ \\
Electron Spectral Index & $\alpha_{e}$ & 2.6 \\
\hline
\end{tabular}




\section{Diffusivity of Injected Protons}

As mentioned in section 1, a run-time analysis of the full, 27-dimensional parameter space of the simulation setup was performed for two reasons: First, to verify the improved performance of the introduced modifications, and second, to examine the respective propagation regime, in which the injected protons reside for a distinct injection energy.

Consequently, the results of the run-time analysis are presented in fig. 2(a), where the monochromatic injection energies of $N_{\mathrm{inj}}^{p}=5 \cdot 10^{3}$ protons obey

$$
E_{0, i}^{p}=10^{4+\frac{i}{2}} \mathrm{GeV} \quad \text { for } i \in\{1,2, \ldots, 11\},
$$

in otherwise similar simulation setups. The plot shows the combined results of ten consecutive runs, with arithmetic mean values of run-times and the standard deviations as uncertainties. Please note that the run-times are normalized to one hundred injected protons. Further, the different data sets refer to two propagation modules of CRPropa, propagationCK and propagationBP, which use different algorithms to solve the equation of motion of a relativistic, charged particle, namely the Cash-Karp and the Boris-Push algorithm, respectively. The latter needed to be modified in order to be used in this particular simulation setup and the data depicted in fig. 2(a) reflects the improved performance when using the propagation $B P$-module.

Two additional effects can be observed in fig. 2(a), besides the performance improvement of propagation $B P$ against propagation $C K$. First, the run-time is lowest for injection energies between $10^{6} \mathrm{GeV}$ and $10^{8} \mathrm{GeV}$. For lower energies, it rises, which can possibly be explained due to the fact that more particles behave rather diffusively than ballistically by smaller gyrations around the magnetic field lines. Recalling the approximation of the gyro-radius of a relativistic particle with charge $q$

$$
r_{g}^{\mathrm{rel}} \approx 3.3 \cdot \frac{E}{\mathrm{GeV}} \frac{v_{\perp}}{c} \frac{|q|}{e}\left(\frac{B}{\mathrm{~T}}\right)^{-1},
$$

yield an estimate for the gyro-radius dependent on the system's parameter, namely energy $E$ in $\mathrm{GeV}$, perpendicular velocity $v_{\perp}$ in terms of the speed of light in vacuum, $c$ and magnetic field strength $B$ in T. Following the argumentation about the diffusivity regime of Reicherherzer et al. in $[10,11]$ and Schlegel et al. in [12], one expects the particles, given enough time, to behave diffusively if the reduced rigidity $\rho$, defined as

$$
\rho=\frac{r_{g}^{\mathrm{rel}}}{l_{c}}
$$

can be found between:

$$
\begin{aligned}
\frac{l_{\min }}{l_{\max }} & \leq \rho \leq 1, \\
0.0039 & \lesssim \rho \leq 1 .
\end{aligned}
$$

Plugging the system's properties energy (eq. (2)), the magnetic field strength $B_{\mathrm{RMV}}$ and the correlation length $l_{c}$ from table 1 yield a (simplified) estimate for the reduced rigidity $\rho$ at the starting 
point of the propagation of the plasmoid:

$$
\begin{aligned}
& \rho \approx \frac{3.3 \cdot 10^{8} \cdot 10^{\frac{i}{2}} \mathrm{~m}}{10^{-2} R}=\frac{10^{-5} R \cdot 10^{\frac{i}{2}}}{10^{-2} R}, \\
& \rho \approx 3.3 \cdot 10^{-3} \cdot 10^{\frac{i}{2}} \quad \text { for } i \in\{1,2, \ldots, 11\} .
\end{aligned}
$$

Consequently, the condition in eq. (5) is met for injection energies lower than $E_{0,4}^{p}=10^{6} \mathrm{GeV}$.

This is partly reflected in fig. 2(a), where the increase in run-time is first observed for $E_{0,3}^{p}=$ $10^{5.5} \mathrm{GeV}$ and energies below. It can possibly be explained due to the relatively short timescale in which the protons reside inside the plasmoid. As mentioned before, at eq. (4), the diffusive regime will be reached only if the particles can perform a certain amount of gyrations before leaving the plasmoid.

Additionally, an increase in run-time can be observed besides the low-energy regime, namely for energies higher than $E_{0,8}^{p}=10^{8} \mathrm{GeV}$. A possible reason for the increase in run-time can be the excessive process load caused by the secondaries.

Figure 2(b) displays the effect of lower injection energies on the ejection time of protons. The ejection time itself is proportional to the time a particle resides in the plasmoid. Here, two simulations are compared, both with a monochromatic injection scenario, one with energy $E_{0,2}^{p}=$ $10^{5} \mathrm{GeV}$ and one for $E_{0,8}^{q}=10^{8} \mathrm{GeV}$.

It can be seen in fig. 2(b) that the peak ejection time is shifted by almost to orders of magnitude for the lower energy. It is worth noticing that regardless of their injection energy, no particle will stay long enough in the plasmoid to meet the boundary condition of a preset maximum trajectory length $D_{\max }=1 \mathrm{pc}$. This fact is reflected by the vertical black dashed line in fig. 2(b), which is the time-converted boundary condition for time-like particles.

\section{Correlation between Gamma-Ray and Neutrino Emission}

The final part of this evaluation aims at answering the question, whether the simulated propagation of the plasmoid along the AGN-jet axis yields a correlated neutrino and gamma-ray emission. The results of this analysis shall be presented in fig. 3(a) and fig. 3(b). First of all, the data was processed without any further restrictions, resulting in the correlation plot as seen in fig. 3(a). Here, no apparent correlation is found, at least not without obtaining huge error margins for the linear regression, which should correlate the number of neutrinos to the number of gamma-rays as

$$
N_{\gamma}=\left(a_{0}+\Delta a_{0}\right) N_{\nu}
$$

where the linear regression eq. (7) was chosen to be calculated without offset due to physical reasons: It is not expected to have a fundamental offset in gamma-ray emission while performing the simulation with the interactions at hand. Nevertheless, the reason for the non-existent correlation is still an open question. It is possible that this is a general phenomenon of the simulation setup or may be related to the low number of photons detected at the plasmoid's surface. It was found that the Breit-Wheeler pair-production module causes the majority of produced photons to interact with the background photon field of the accretion disk, which illuminates the plasmoid. Such a 




(a)

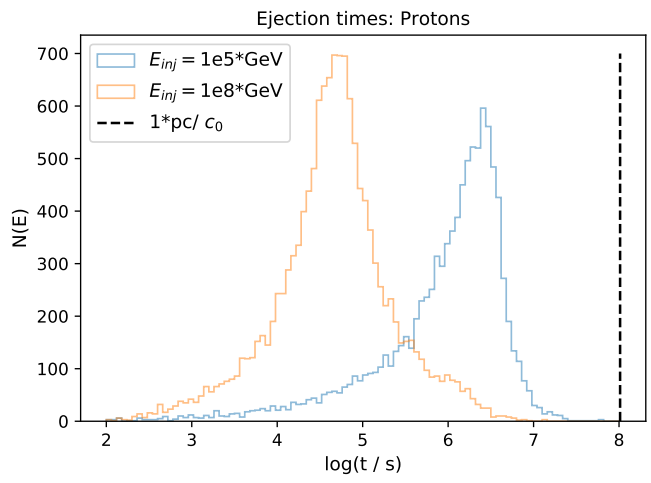

(b)

Figure 2: Panel (a): Run-time analysis for the different propagation modules, propagationCK and propagationBP, performed on 32 threads for the parameter combination. The overall time consumption is significantly higher for lower injection energies in both modules, with an overall better performance of the module propagationBP.

Panel (b):Comparison: Ejection times of protons for $N_{p}=1000, E_{p}=10^{5} \mathrm{GeV}$ and $E_{p}=10^{8} \mathrm{GeV}$. The effect of a lower injection energy on the ejection time can be seen. The vertical black dashed line marks the boundary condition of a maximum trajectory length $D_{\max }=1 \mathrm{pc}$. The propagationBP module was used in both simulations.

scenario could explain a potential anti-correlation between gamma-rays and neutrinos: gamma-rays are absorbed, while neutrinos escape.

As a cross-check, a different simulation setup was designed where the Breit-Wheeler pair-production is disabled in order to compare the effect of the missing $\gamma \gamma$-attenuation with fig. 3(a). The analysis of the new simulation is shown in fig. 3(b). In this case, a direct correlation of neutrino and gammaray emission is found when calculating the coefficients of the linear regression eq. (7). However, there is no obvious, physical scenario imaginable in which the accretion disk photons are able to interact with the primary protons but not with the secondary gamma-rays. Hence, fig. 3(b) serves only as an illustration. 


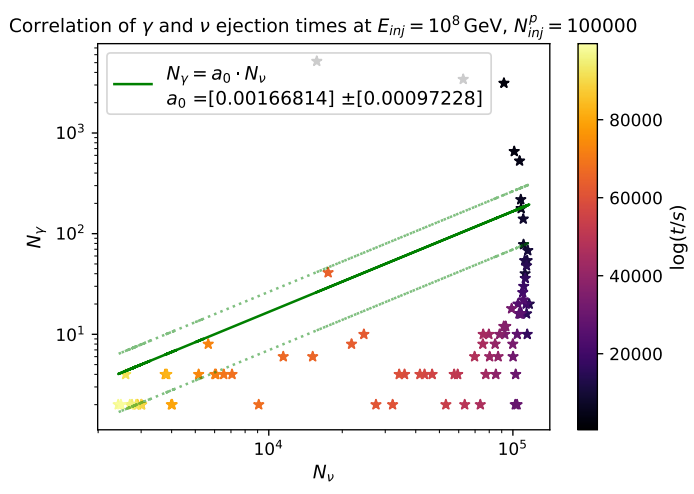

(a)

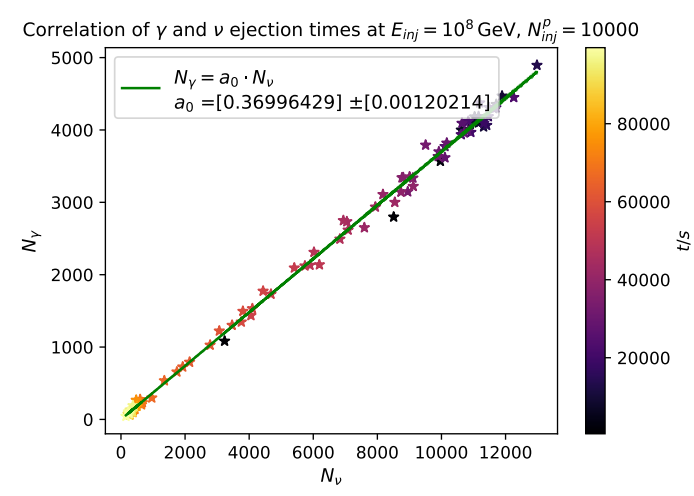

(b)

Figure 3: Panel (a): The correlation between neutrino and gamma-ray emission at equal points in time, which are color-coded by the bar on the right-hand side. Please note the double-logarithmic scale of the axis, which was enabled to take the low photon sample size into account. Coefficients and errors of the linear regression are computed using the scipy-routine optimize.curve_fit. As it is apparent in this figure, no correlation can be observed without obtaining huge error margins. This is partly explained due to a small sample size of photons for the chosen time steps, which may be explained by the impact of the invoked $\gamma \gamma$ attenuation of Breit-Wheeler pair-production between produced photons and thermal accretion disk photons. Panel $(b)$ : The same simulation setup and processing as for panel (a), but with disabled Breit-Wheeler pair-production module. Here, a correlation between gamma-ray and neutrino emission is found. 


\section{References}

[1] Astrophysical neutrinos and cosmic rays observed by IceCube, Aartsen, M. G. et al., Advances in Space Research, 2018

[2] Synchrotron emission from shock waves in active galactic nuclei, Biermann, P. L. and Strittmatter, P. A., ApJ, 1987

[3] Note on high-energy neutrinos from active galactic nuclei cores, Stecker, F. D., PRD, 2005

[4] Neutrino production through hadronic cascades in AGN accretion disks, Nellen, L. and Mannheim, K. and Biermann, P. L., PRD, 1993

[5] High-energy neutrinos from extragalactic jets, Mannheim, K., Astroparticle Physics, 1995

[6] Blazar flares as an origin of high-energy cosmic neutrinos? Murase, K. and Oikonomou, F. and Petropoulou, M., ApJ, 2018

[7] Closing in on the origin of Galactic cosmic rays using multimessenger information, Becker Tjus, J. and Merten, L., Physics Reports, 2020

[8] On the relative importance of hadronic emission processes along the jet axis of Active Galactic Nuclei Hoerbe, M. R. and Morris, P. J. and Cotter, G. and Becker Tjus, J. MNRAS, 2020

[9] CRPropa 3.1 - a low energy extension based on stochastic differential equations Merten, L. and Becker Tjus, J. and Fichtner, H. and Eichmann, B. and Sigl, G. JCAP, 2017

[10] Turbulence-level dependence of cosmic ray parallel diffusion Reichherzer, P. and Becker Tjus, J. and Zweibel, E. G. and Merten, L. and Pueschel, M. J. MNRAS, 2020

[11] Regimes of cosmic-ray diffusion in Galactic turbulence Reichherzer, P. and Merten, L. and Dörner, J. and Becker Tjus, J. and Pueschel, M. J. and Zweibel, E. G. arXiv e-prints, 2021

[12] Interpolation of Turbulent Magnetic Fields and Its Consequences on Cosmic Ray Propagation Schlegel, L. and Frie, A. and Eichmann, B. and Reichherzer, P. and Becker Tjus, J. ApJ, 2020

[13] Cosmic Neutrinos from Temporarily Gamma-suppressed Blazars Kun, E. and Bartos, I. and Becker Tjus, J. and Biermann, P. L. and Halzen, F. and Mezó, G., ApJ, 2021 